\title{
RESEARCH AND DEVELOPMENT IN THE FIELD OF PASSIVE COMPONENTS
}

\author{
D. S. CAMPBELL \\ Electrical Engineering Dept., Loughborough University, Loughborough, Leics., U.K.
}

(Received December 21, 1973)

\begin{abstract}
This paper firstly examines the market situation in passive components in relation to the total position of the electronics industry. Expenditure on research and development is then discussed and examples given of recent and present research and development activities in the field of passive components. The situation with regard to the publication position on research and development in passive components is briefly considered and it is concluded that there is a positive need for a journal covering the passive component field and acting as an outlet for papers generated in this area.
\end{abstract}

\section{INTRODUCTION}

This paper aims to give a brief analysis of research and development in the field of passive components. The analysis is firstly in terms of the market situation from which is deduced which component areas are associated with the major part of the turnover. Secondly, the expenditure on research and development in all passive components areas is discussed and finally examples are given for the important components of recent and present research and development activities.

The market situation has been analysed in terms of statistics for the U.K. covering up to the end of 1972. Although details will be slightly different, the picture will in the main be the same with regard to Europe but with a multiplication factor of approximately 3 for all the figures. A similar assumption can be made for the total of the U.S.A., Canada, Australia, Japan etc., but in this case with a multiplication factor of $x$ 10.

\section{MARKET ANALYSIS}

Market statistics are given in various publications. Such figures quote turnover in terms of manufacturers' sales ex-works and do not include re-export figures. Also, values are all given at current prices and no adjustments are made for inflation.

One such set of statistics are those published by the National Economic Development Organisation. ${ }^{1}$ These figures give both a general and specific break-
TABLE 1

Total U.K. sales of equipment or components. 1971 and 1972 (fM)

\begin{tabular}{lcc}
\hline & 1971 & 1972 \\
\hline Telecommunications & 293 & 320 \\
Consumer goods & 214 & 310 \\
Capital equipment & 242 & 253 \\
Passive components & 210 & 250 \\
Computers & 205 & 220 \\
Active components & 129 & 177 \\
& 1,297 & 1,521 \\
\hline
\end{tabular}

down of the market situation in the electronics industry.

The general picture presented with regard to sales is summarised in Table I. These figures show a total increase of $17 \%$ over the values for 1971 and this represents a considerable recovery after 1971 , when the industry only achieved a growth of $8 \%$ from 1970 to 1971 . Moreover, since these figures became available, all the indications are that the increase in sales has continued through 1973.

It can be seen from Table I that the component market in 1972 as a whole, represented $28 \%$ of the total electronics industry with passive components themselves being associated with just under $18 \%$.

The figures given in Table I can be examined in further detail, however, to determine the position in specific areas. The picture over the period 1969 to 1971 with regard to the passive and active component market is shown in Figure 1. This shows a steady 


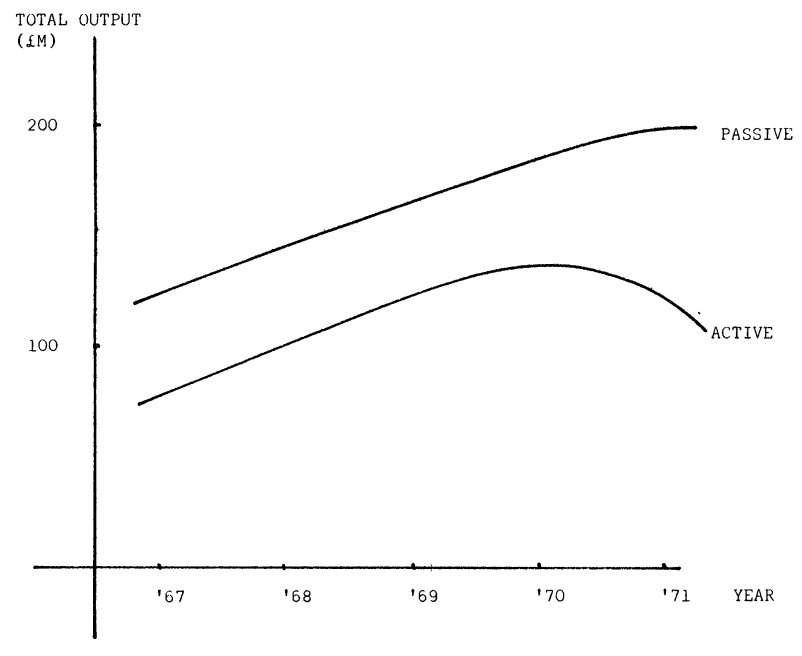

FIGURE 1 Total output of passive and active components as a function of time.

increase in both areas up to 1970 but with a fall-off in the active component market after this date, almost certainly due entirely to price erosion rather than to a fall-off in numbers of devices produced. If one now examines these curves in still more detail, it is found possible to split the active and the passive component areas into separate device regions. Such a split is shown for active devices in Figure 2, where it can be seen that in 1972 valves accounted for over

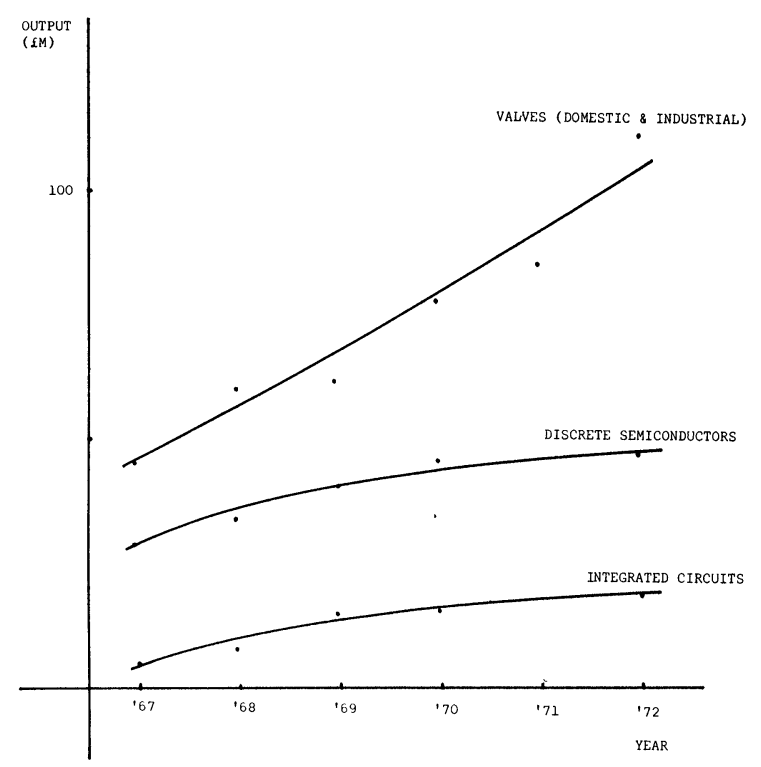

FIGURE 2 Output of classes of active components as a function of time. half of the turnover in the active component industry. Such a situation is probably explained by the advent of colour television and hence the manufacture of colour television tubes in large quantities, and this view is confirmed by an analysis that can be undertaken on the valve market itself. This shows that between 1971 and 1972, turnover in the domestic valve market increased from $£ 60 \mathrm{M}$ to $£ 82 \mathrm{M}$, whereas that in the industrial valve market, including Magnetrons, Klystrons etc., only increased from $£ 25 \mathrm{M}$ to $£ 28 \mathrm{M}$.

A similar examination can be made for passive components and the results are shown in Figure 3. Figures are only available in this case for the four years from 1968 to 1971 . The curves shown in Figure 3 relate to the top six products in the passive component field and include not only items such as fixed capacitors, fixed resistors etc., but also turntables. It may appear strange to classify gramophone turntables as passive components but this is the classification given in the Government statistics available. It is, in fact, the considerable increase (42\%) from 1970 in the area of turntables that results in a higher figure for passive component turnover in 1971 than in 1970. All the other areas show no growth after inflation is allowed for and in fact, in some areas such as fixed capacitors and fixed resistors, there has been a considerable fall-off. However, evidence for the period 1972 to 1973 indicates a substantial reversal of these trends.

It is useful to analyse the passive component market in a little more detail and Table II shows the

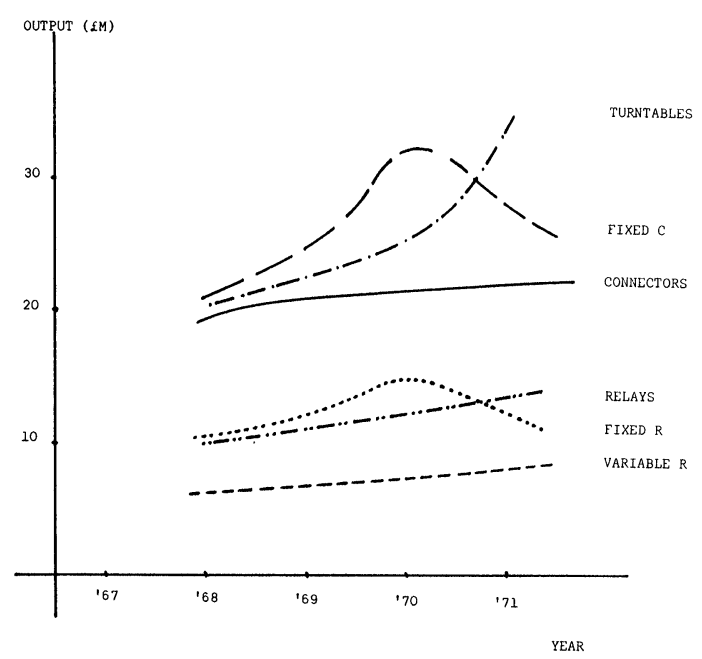

FIGURE 3 Output of classes of passive components as a function of time. 
TABLE II

Sales of passive components in 1970 and 1971

\begin{tabular}{lrr}
\hline & 1970 & 1971 \\
\hline Turntables & 24.3 & 34.4 \\
Fixed C & 30.8 & 28.3 \\
Connectors & 21.9 & 22.2 \\
Relays & 12.4 & 13.6 \\
Fixed R & 15.3 & 12.2 \\
Variable R & 9.4 & 9.0 \\
Magnetic materials & 7.4 & 8.5 \\
Terminals & 7.2 & 7.6 \\
Transformers, chokes etc. & & \\
(Power) & 4.2 & 7.1 \\
Transformers, chokes etc. & & \\
(R.F. etc.) & 6.9 & 6.2 \\
Switches & 5.0 & 5.8 \\
Ferrites & 3.7 & 4.0 \\
P.C. Boards & 4.0 & 3.8 \\
Quartz crystal devices & 4.8 & 3.5 \\
Valve holders etc. & 1.4 & 1.4 \\
Thick and thin films, hybrids & 0.7 & 1.2 \\
Variable C & 1.2 & 1.1 \\
\hline
\end{tabular}

sales of passive components for 1970 to 1971 , for not only the components shown in Figure 3, but for the top 17 categories. This table shows that good growth has been obtained in 3 product areas. These categories are turntables, power transformers, where the increase over 1970 has been $69 \%$ and finally, hybrid thick and thin film circuits, where the increase has been $72 \%$. In this last category further figures for 1972 have shown an increase to approximately $£ 4 M$ and predicted figures for 1973 are $£ 6 \mathrm{M}$.

In summary, an analysis of market figures available shows that there is considerable industrial involvement in the manufacture of both active and passive components. In both of these areas there are particular components showing considerable growth in output.

\section{RESEARCH AND DEVELOPMENT EXPENDITURE}

Given the market analysis that has now been discussed, the question to be asked is whether or not this would allow one to make an estimate of the amount of Research and Development activity involved in passive component manufacture. Help can be obtained in such an analysis from the use of obsolescence rate curves (Refs. 2, 3 and 4). Such curves relate the percentage of turnover that has to be spent on research to keep the industry in a viable state, but without considerable expansion, to the

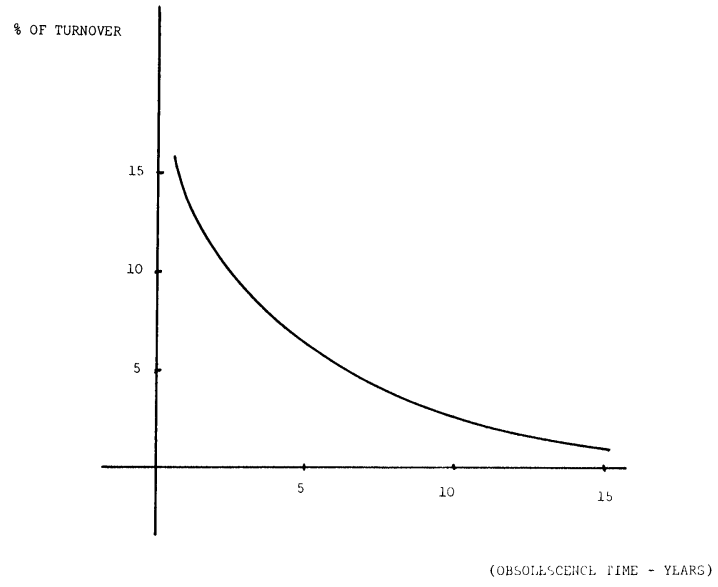

FIGURE $4 \%$ of turnover that should be spent on research as a function of the obsolescence time of the product.

obsolescence time of the product in years. Unfortunately, such curves are open to considerable discussion, but a typical curve that has been suggested is shown in Figure 4.

For the passive component industry, an average obsolescence period of 10 years appears to be a fair compromise, as there are very few components that will not have been modified in some way during that period, so that one will have today a product that is improved in terms of size, cost and reliability over that manufactured 10 years ago. Such a figure gives a research expenditure of $3 \%$ of turnover to keep the industry in a viable state.

Covered by this $3 \%$ expenditure are both basic and applied research. Basic research is defined as work which has no specific commerical objective, whereas applied research has a general or particular objective associated with a product. It should also be noted that this figure of $3 \%$ does not include development expenditure which would be running at at least 5 times the rate for basic and applied research expenditure and would cover the introduction or improvement of specific products including pilot plant activity.

Such an analysis applied to the total passive component market would give an expected research expenditure in the U.K. of approximately $£ 6 \mathrm{M}$ in 1971 and a development expenditure of £30M.

Unfortunately, it is not possible to determine whether these figures have been realised for the passive component industry from the data published by N.E.D.O. ${ }^{1}$ These data do, however, include figures for the electronics industry as a whole. Table III gives these figures together with theoretical figures for 
TABLE III

Total research and development in the electronics industry for 1971 compared with calculated value ( $(\mathrm{M})$

\begin{tabular}{llll}
\hline & Turnover & Research & Development \\
\hline Available figure & 1428 & 30 & 115 \\
Calculated figure & - & 43 & 190 \\
\hline
\end{tabular}

research and development expenditure, assuming a 10 year obsolescence period and with development running at 5 times research expenditure. The published figures show a lower value of basic and applied research expenditure and of development, than those expected from Figure 4. This would lead one to estimate that the probable research expenditure in the passive component industry for 1971 was actually running at $£ 3 \mathrm{M}$ and the development expenditure at $£ 15 \mathrm{M}$.

It should be noted that a decrease in the figures from those expected from Figure 4 could be associated not only with a difference in the average obsolescence time in the products from the assumed value of 10 years, but also with the effect of individual company policies on research and development itself. An acquisition policy practised by a company would obviously reduce the amount of research and development expenditure actually used for the employment of scientific staff and the production of pilot plant machinery and substitute instead, expenditure on the acquisition of stock and shares. These latter figures would not appear in the present analysis so that although a company may well be progressing rapidly by a policy of acquisition, its research and development expenditure could be substantially lower than that indicated.

\section{AREAS OF RESEARCH}

In order to illustrate some of the research and development activities that have and are taking place in the passive component field, some component types can be examined in more detail. Not all of these types are in the top groups of Table II, but the rate of growth is often as important as total turnover in assessing the importance of an activity.

Even for components in the top groups, details are not always available because of the commercial nature of the work. This is particularly true for the area of turntables, where competition is severe, but where development advances are still being made (e.g. Bang and Olufson's Tangential tracking systems for record playing equipment ${ }^{5}$ ).

\subsection{Fixed Capacitors}

As with all components, miniaturisation coupled with cost saving are the important criteria. New plastic materials ${ }^{6}$ are being currently exploited as capacitor dielectrics and materials such as polypropylene ${ }^{8,9}$, polycarbonate ${ }^{7,8}$ and polysulphone ${ }^{10}$ are being used. All these materials are available in thin sheets with thicknesses in some cases as low as $2 \mu \mathrm{m}$, and they also exhibit low dielectric loss (in the case of polysulphone, up to a temperature of at least $150^{\circ} \mathrm{C}$ ). Electrical conduction processes in these materials are now under investigation, particularly with regard to causes for variability in electrical behaviour.

Not only are these plastics under thorough investigation, but the search is continually being made for newer materials which have even better or different characteristics. One such material, polyvinylfluoride, has interesting piezoelectric properties, ${ }^{8}$

In aluminium electrolytic capacitors, the use of higher gain foil has resulted in considerable savings in aluminium and hence in the space required for a particular capacitance value. Studies of etching characteristics are being undertaken at various establishments, with a view to increasing this gain still further. ${ }^{11}$ In association with such studies, the impedance behaviour of electrolytic capacitors is now understood in some detail ${ }^{12}$, and is of importance in determining the maximum superimposed alternating current (ripple rating) that an electrolytic capacitor can accept without overheating.

In the case of tantalum electrolytics, some of the present emphasis is associated with the use of different powders ${ }^{13}$ to produce higher surface areas and hence increase the capacitance available per gram of sintered powder. A further development in electrolytic capacitors is that associated with solid aluminium, a term which has been used for two different areas of investigation. One type is concerned with the replacement of the wet electrolyte in a conventional etched foil aluminium capacitor with a solid material based on manganese oxide ${ }^{14}$. However, another line of attack is concerned with the preparation of sintered aluminium blocks in an analogous manner to those formed from tantalum powder ${ }^{15}$.

\subsection{Connectors}

The design of connectors has been continually advancing with regard to solving problems associated with printed circuits boards. hybrid microelectronics and integrated circuitry and a wide variety of connectors is now available. ${ }^{16}$ Research work is 
associated with obtaining satisfactory connecting points in the connector with cost being the main criterion. Metallurgical investigations of this problem are in hand at various centres (e.g. Ref. 17). Studies are associated with the use of selective plating in the contact area or the possible use of oxidisable materials that will give hard areas of oxide which will penetrate any tarnish formed during use ${ }^{18}$.

\subsection{Relays}

Relays continue to be widely used and new types are still under development. A recent article shows that it is possible to replace semi-conductors with relays, ${ }^{19}$ a reversal of the normal approach one sees these days. Research work in relays, as in the case of connectors, is associated with metallurgical studies to find materials that will neither tarnish, nor act as catalysts for the decomposition of organic vapours present in the atmosphere. Transfer studies still continue on devices such as reed relays ${ }^{20}$, with the aim of determining the causes of tranfer of material from one contact to the other in D.C. relays, a situation which can become serious if millions of operations are required.

\subsection{Fixed and Variable Resistors}

Basic and applied research in the area of resistors is often associated with obtaining an understanding of the electrical conduction processes in the materials. Modern materials, particularly of the thick film type, are often of a composite nature with metal or metal-oxide particles separated from one another by a glass or plastic dielectric medium ${ }^{21-23}$. Studies on these structures are being undertaken at various centres such as Chelsea College (London University). More conventional resistors based on the use of low temperature coefficient resistance alloys are now readily available as a result of metallurgical studies on alloys. It is now possible to use metallic structures that will give a temperature coefficient as low as $1 \mathrm{ppm} /{ }^{\circ} \mathrm{C}$ for resistor values up to $100 \mathrm{~K} \Omega^{24}$.

\subsection{Suppression}

Although not an area shown directly in Table 2, the field of suppression, involving as it does, combinations of resistance, capacitance and inductance, is now becoming increasingly important because of the need to prevent the pollution of the electrical environment by various equipments, professional, industrial or domestic. ${ }^{25}$ Studies have included an examination of the filtering characteristics obtained from various components ${ }^{26}$, particularly with reference to the use of wound components which have both inductance and capacitance. Also, studies are currently under way in the field of noise generation in order to determine the most satisfactory method of suppression (e.g. studies on harmonics and thyristor noise $^{27}$ ).

\subsection{Hybrid Circuits}

As has been previously noted, there has been a very rapid growth in the activity connected with thick film and hybrid circuits. The extent of this activity is illustrated by the scientific meetings now being held on this subject, e.g. the Conference on Hybrid Microelectronics held at the University of Kent at Canterbury in the U.K. (See Ref. 21,22,29 as well as the general texts available, Ref. 28). As well as problems associated with materials which have already been referred to, studies are going on in the area of adhesion and the bonding of discrete devices to thick film circuits ${ }^{29}$. Although a considerable amount of research and development effort in this area is associated with applications, nevertheless, component type problems are also under study in the area of thermal design, failure and reliability.

\subsection{Piezoelectrics}

Piezoelectric ceramics have been developed to a stage where they provide a class of materials suitable for many transducer applications ${ }^{30}$. Development work in this area still continues both with regard to the materials themselves and also the type of transducer that is required for specific applications. An encouraging development in this area is the possible use of piezoelectric and ferroelectric materials for adaptive memory devices ${ }^{31}$.

Basically an application of a piezoelectric material, and also closely associated with thick film technology, is the surface acoustic wave device. An understanding of the behaviour and uses of these devices is under considerable study both with regard to materials based on quartz or lithium niobate ${ }^{32}$, or the possible use of a non-piezolectric medium ${ }^{33}$, associated with a separate transducer ${ }^{34}$. The use of these devices in association with hybrid microelectronic techniques has recently been discussed by Lambert et al. ${ }^{35}$ 


\section{PUBLICATIONS}

It would appear, as discussed above, that an expenditure of around $£ 3 \mathrm{M}$ on basic and applied research in passive components has obtained in the U.K. in 1971. If one considers the total expenditure in Europe, U.S.A., Canada and Japan, a total of $£ 40 \mathrm{M}$ is a reasonable estimate. With regard to development, not all work is of a publishable nature since it may be very closely associated with pilot plant activities etc., i.e. projects which have considerable commercial security. If, however, one assumes that a quarter of development expenditure is associated with publishable long-term objectives, then the total expenditure for which publishing outlets are required in the passive component field could be around $1100 \mathrm{M}$ per annum.

A lot of this publishing will naturally occur in specialised journals associated with particular areas of activity, e.g. Electrochemistry, Magnetic Materials etc. However, it can be argued that there is a positive need for a journal covering the passive component field and acting as an outlet for papers generated by a significant proportion of the $1100 \mathrm{M}$ expenditure mentioned above.

\section{CONCLUSIONS}

This paper has examined the market position in the field of passive components and has briefly summarised some of the activities taking place in the more important areas in this field. It is concluded that there is considerable and world-wide expenditure on basic and applied research and long-term development for which a suitable publication forum is required.

\section{REFERENCES}

1. Annual Statistical Survey of the Electronics Industry. H.M.S.O. October, '73.

2. D. S. Campbell, "New materials in the capacitor industry'. Proc. 2nd Int. Conf. on Product Dev. \& Man. Tech. (Ed. D. S. Ross) pp. 86 - 104. (1972).

3. E. A. D. White, 'The effective use of research and development funds in the U.K. electronic industry'. Industrial Research Budgeting Project. Elec. Eng. Dept., Imperial College, London. 1971.

4. W. E. Duckworth, New Scientist 32 No. 524. p. 564. (1966).

5. C. J. Myers, 'Domestic science'. Physics Bulletin 24 pp. 350-352. (1973).

6. W. Goldie, Metallic Coating of Plastics Vol. 2 (Electrochemical Publications. U.K.). 1969.
7. P. D. Habermel, 'Plastic film capacitors'. Radio \& Elec Eng. 40 pp. 259-264. 1970.

8. P. J. Harrop, Dielectrics (Butterworths) 1972.

9. P. J. Harrop,'The use of polypropylene in capacitors'. Electronic Components 16 pp. 14-17 Jan. 1st, 1974.

10. R. K. Walton,'Polysulphone in the electronics industry'. Proc. Elec. Components Conf. pp. 130-134. 1968.

11. D. S. Campbell, 'Electrolytic capacitors'. Radio \& Elec. Eng. 41 pp. 5-16. 1971.

12. A. R. Morley and D. S. Campbell, 'Electroly tic capacitors - their fabrication and the interpretation of their, operational behaviour'. Radio \& Elec. Eng. 43 pp. 421-429. 1973.

13. L. F. Harris, 'Trends in the design and performance of tantalum capacitors'. Electrocomp. Sci. Technol. 1, p. 11.

14. B. Gondswaard, 'Solid aluminium electrolytic capcitors, 121-series'. Mullard Tech. Comm. 111 pp. 30-36, July, 1971.

15. W. J. Bernard and E. J. Fresia 'Anodic oxidation of porous aluminium pellets' Electrocomp. Sci. Technol., 1, p. 59.

16. Connectors feature in Electron No. 41 pp. 27-43. 6th Dec., 1973.

17. P. M. A. Sollars, 'Wire wrapped joints - a review'. Electrocomp. Sci. Technol., 1, p. 17

18. D. Pedder, Private communication. 1973.

19. D. Trowbridge, 'Replacing semi-conductors with relays'. Electronic Components 14 p. 517 (1973).

20. T. A. Davies, 'Transfer of metal between light duty electrical contacts'. J. Mat. Sci. 3 pp. 314-320 (1968).

21. B. Walton, 'Principles of thick film materials formations' Proc. Conf. on Hybrid Microelectronics. I.E.R.E. Conf. Proc. No. 27, pp. 39-46 (1973).

22. M. V. Coleman, 'Evaluation methods for the examination of thick film materials' Proc. Conf. on Hybrid Microelectronics. I.E.R.E. Conf. Proc. No. 27. pp. 221-238. 1973.

23. R.W. G. Rose, 'An introduction to conductive plastic potentiometers'. Electron No. 30. pp. 40-41. (14th June, 1973).

24. D. R. Bell, 'Wirewound general purpose and precision resistors'. Radio \& Elec. Eng. To be published.

25. P. J. Harrop, 'Interference suppression'. Electron No. 13. pp. 13-14 (11 th Han., 1973).

26. $\mathrm{H}$. Kaiserwerth, ' $\mathrm{RF}$ interference suppression chokes for thyristor devices' Siemens Electronic Comp. Bulletin VII pp. 49-52. No. 3. (1972).

27. J. K. Hall and N. C. Jones, 'The frequency spectrum generated by thyristor control'. Electrocomp. Sci. Technol., 1, p. 43.

28. G. Planar and L. S. Phillips, Thick Film Circuits' (Butterworths, London). 1972.

29. M. G. Harwood, J. Schofield, and M. V. Burrell, 'Conducting Polymers for die bonding of semi-conductors to thick film circuits' Proc. Conf. on Hybrid Microelectronics. I.E.R.E. Conf. Proc. No. 27 pp. 63-70. (1973).

30. I. Flinn, 'Piezoelectric ceramics'. Electron No. 32 pp. 59-64. (12th July, 1973).

31. W. H. Dennis 'Ceramic ferroelectrics' Electron No. 41 pp. 49-52 (6th Dec., 1973).

32. E. Dienkesaint and P. Hartemann, 'Acoustic surface wave filters'. Ultrasonics pp. 24-30. (January, 1973).

33. C. P. Sandbank and M. B. N. Butler, 'Acoustic surface waves on isopaustic glass'. Electronic Letters 7 pp. 499501. (1971). 
34. D. R. Evans, M. F. Lewis, and E. Patteson, 'Sputtered $\mathrm{ZnO}$ surface wave transducers'. Electronic Letters 7 pp. 557-558. (1971).

35. R. D. Lambert, P.M. Grant, D. P. Morgan and J.H.
Collins, 'Programmable surface acoustic wave devices utilising hybrid microelectronic techniques' Proc. Conf. on Hybrid Microelectronics. I.E.R.E. Conf. Proc. No. 27. pp. 161-171. (1973). 

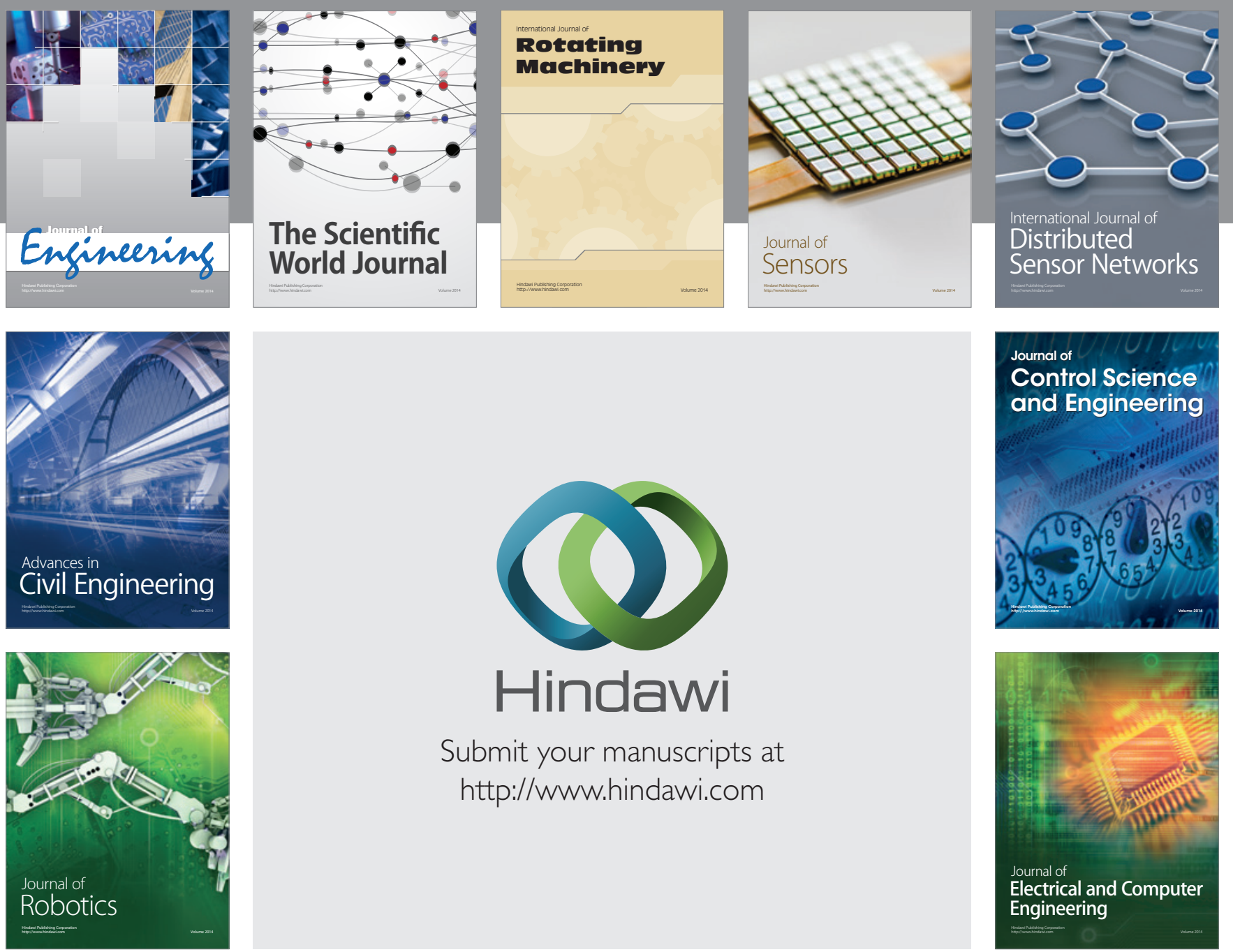

Submit your manuscripts at

http://www.hindawi.com
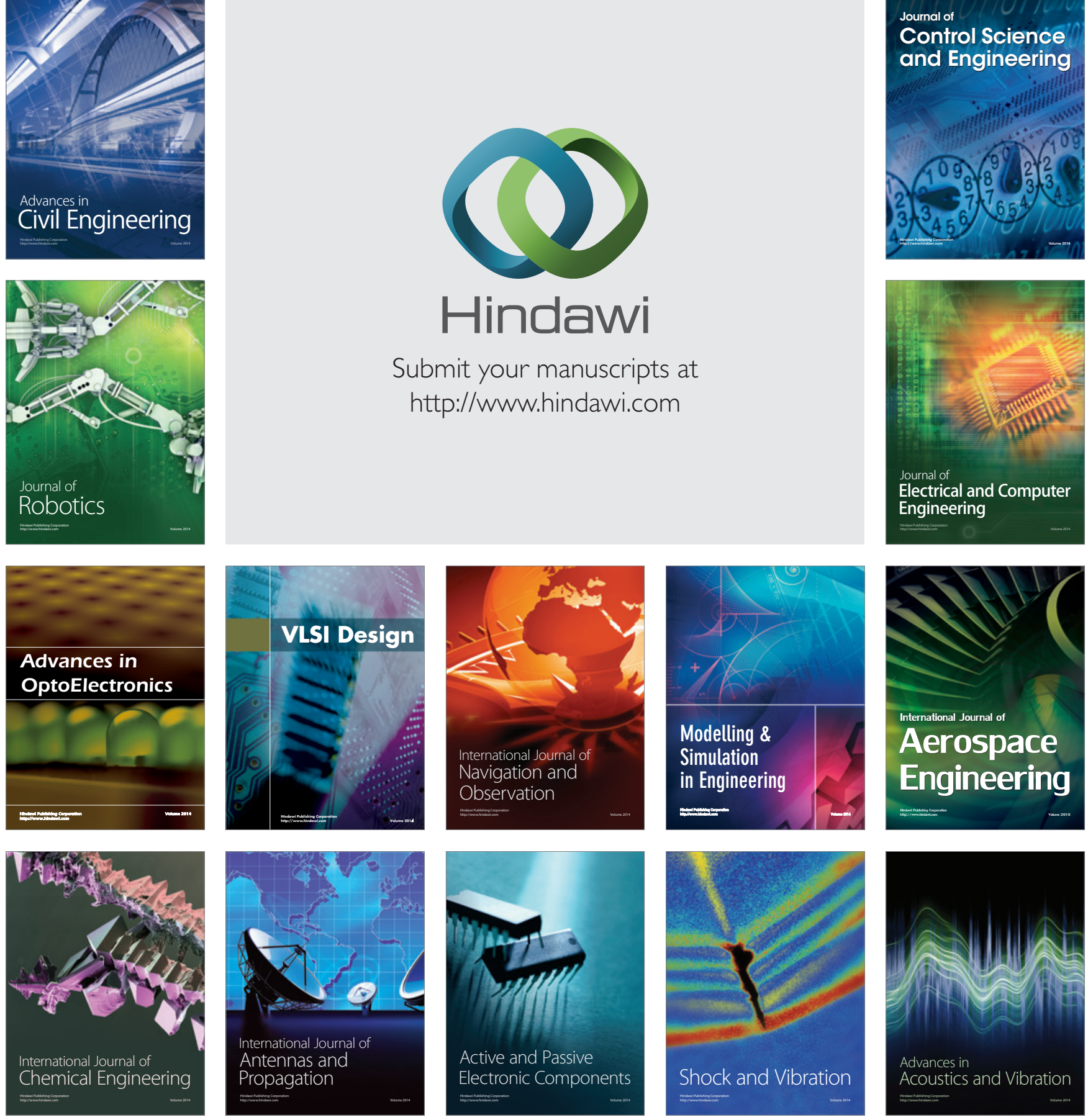